\title{
Influence of Technological Factors on Sensitivity of Analytical Devices Based on Surface Plasmon Resonance
}

\author{
Glib Dorozinsky, Tamara Doroshenko, Volodymyr Maslov* \\ Department of Physics and Technological Bases of Sensory Materials, V. Lashkaryov Institute of Semiconductor \\ Physics NAS of Ukraine, Kyiv, Ukraine \\ Email: vpmaslov@ukr.net
}

Received 5 May 2015; accepted 31 May 2015; published 3 June 2015

Copyright (C) 2015 by authors and Scientific Research Publishing Inc.

This work is licensed under the Creative Commons Attribution International License (CC BY). http://creativecommons.org/licenses/by/4.0/

c) (i) Open Access

\begin{abstract}
Considered in this paper are the factors influencing sensitivity of analytical devices based on surface plasmon resonance phenomenon. The decrease in temperature influence on sensitivity of these devices can be reached using thermo stabilization of the whole device with flow cells for studied substances. Adduced here are some results concerning the influence of deposition geometry for nano-dimensional gold layer covering the surface of operating element on sensitivity of these devices.
\end{abstract}

\section{Keywords}

Deposition Technology, Optical Sensor, Surface Plasmon Resonance, Sensitivity of Devices

\section{Introduction}

Determining the concentration of chemical and biological substances in various media is of great importance and has attracted researchers' attention in relation with development of such branches as pharmacology, medicine, food industry, and control of ambient medium. Development of investigations for creation of reliable analytical devices capable to detect small amounts of above substances should result in efficient solutions of these tasks. Among these devices, chemical and biological sensors, which are analytical systems containing a layer of biological or chemical molecules and some physical transducer joined with it, take an important place. Sensitivity and accuracy of results obtained with these analytical devices depend both on characteristics of the sensitive layer and parameters of the physical transducer.

"Corresponding author.

How to cite this paper: Dorozinsky, G., Doroshenko, T. and Maslov, V. (2015) Influence of Technological Factors on Sensitivity of Analytical Devices Based on Surface Plasmon Resonance. Journal of Sensor Technology, 5, 54-61.

http://dx.doi.org/10.4236/jst.2015.52006 
One of the promising directions in developing analytical devices is application of surface electromagnetic waves from the optical range in 1D-3D nanostructures as well as observation of resonance phenomena arising in this case (for example, surface plasmon resonance-SPR). Diagnostically facilities based on the SPR phenomenon possess high sensitivity to low concentrations of studied substances, which allow using them not only as gas analyzers but also as precise analytical devices for laboratory investigations in food, chemical, pharmaceutical industry, in agriculture, medicine, and ecology [1] [2].

SPR occurs in a thin metal film with a negative dielectric permittivity and high electric conductivity. This film is located on a transparent dielectric substrate and is a sensitive element. The SPR phenomenon lies in the fact that for the angles of radiation incidence on the thin metal film, which correspond to total internal reflection at the boundary "film-substrate", conduction electrons in the metal film are excited by monochromatic and coherent laser radiation.

This excitation is observed as a sharp drop in the intensity of reflected light for some definite angle of incidence of laser radiation. It is the so-called "resonance angle". For analytical instrumentation engineering, it is important that its value depends on the refractive index and concentration of analyzed substance on the surface of the sensitive element that is in contact with the studied liquid or gas medium. Most often used as the sensitive layer is gold being the metal with high electric conductivity and high chemical inertness.

The sensitivity of detection of refractive index changes is defined by the least angle shift of the SPR minimum, which can be reliably determined. This minimal shift corresponds to a minimal registered change in the refractive index value. To estimate the sensitivity, a number of producers turning out the devices based on SPR phenomenon use the value of this minimum shift in the position of the SPR curve in relative angle units RU (RU = $8.3 \times 10^{-5}$ angular degree, which is equivalent to one picogram of substance on the surface of SPR sensor), while other producers measure the sensitivity in absolute values of the refractive index RIU (refractive index units) [3]. In these cases, changes in the resonance angle are in proportion to those in the concentration of substance in the analyzed volume.

Therefore, in liquids this sensor reacts only on changes in indexes of refraction and absorption of the studied medium. In gas medium, one can observe adsorption of impurity components on the sensor surface, which results in increasing the concentration in the sensitive zone as compared with that in the rest volume. Also, this sensor reacts both on changes in index of refraction (absorption) and in the thickness of adsorbed layer on the surface of sensitive element. The adsorption degree depends on material and surface properties (roughness, porosity and so on). The adsorption degree depends on material and surface properties (roughness, porosity and so on). The increase in surface porosity of sensitive element enables to enhance the sensor response when analyzing both gas media [4] [5] and water solutions [6].

The sensitivity of SPR method is also defined by the width and shape of resonance curve, which define the accuracy of determining the position of its minimum. Therefore, one of possible ways to considerably increase (by several orders) the sensitivity of SPR sensors lies in narrowing the resonance spectrum. It can be attained using special diffraction or holographic structures on the sensitive area of sensors [7]. Narrowing the resonance spectrum can be also reached using bimetal layers "silver-gold" on the sensor surface [8] as well as by increasing the wavelength of laser radiation exciting SPR [9].

Evolution of the sensitivity increase in SPR devices can be represented as follows. In 1998, the firm BIACORE reported about the reached sensitivity $2 \times 10^{-5} \mathrm{RIU}$, and three years ago the sensitivity was improved to $1 \times 10^{-5}$ RIU. At the same time, other authors [10] reported the value $1.2 \times 10^{-6}$ RIU. In 2014, the firm BIACORE reported the attained value $3 \times 10^{-8}$ RIU, while the firm Reichert reached the sensitivity $1 \times 10^{-7}$ RIU [11].

The further increase in the sensitivity is limited by frequency stability and the spectral width of semiconductor laser radiation [12], photo detector noises and thermal drift of facilities. The decrease in the temperature influence on the SPR device sensitivity can be provided using thermal stabilization not only of the sensitive element but also of the whole device with flow cells containing the studied substances [13]. Besides, as a result of experimental thermo-vision investigations, there were suggested optimized temperature regimes for device operation [14].

The increase in the sensitivity of SPR devices can be also reached by modification of the surface of sensitive element as well as by optimization of technology for manufacturing them. In dependence of the solved specific task, the simplest way is to use highly selective sorbents or their mixtures deposited onto the sensitive surface.

The purpose of this work is to study the influence of changes in geometry of deposition of the layer where plasmon is excited on the sensitivity of a device based on SPR. 


\section{Materials and Methods}

Investigations were carried out to optimize deposition geometry for bimetal layers on the surface of sensitive elements. As material for layers, we used gold. The layers were deposited step-by-step on the substrate from $\Phi 1$ glass in the vacuum chamber of ВУП-5M facility under the pressure $1 \times 10^{-3} \mathrm{~Pa}$ (Figure 1). We observe three samples. Deposition of the first layer with the thickness $35 \mathrm{~nm}$ was performed on the substrate located in parallel to the surface of evaporator (typical scheme), while the second layer was deposited onto the first one located at some angle relatively to the evaporator. We used two deposition angles: 45 and 70 degree.

The thickness of the second layer after angular deposition was approximately 30 and $17 \mathrm{~nm}$ for deposition angles 45 and 70 degree, respectively. The thickness of the deposited layer was determined using the quartz crystal thickness-meter with account of changes in the frequency of its vibration.

Used in this experiment were two types of sensitive elements, namely, type 1-prepared by typical deposition with the gold film thickness $35 \mathrm{~nm}$ and type 2-with angular deposition geometry (two samples). Experiments were performed using Multiple Mode that allowed changing the angle of laser beam incidence on the spectrometer sensor element within 17 degrees for 2 seconds. In this mode, every scan enabled to determine and register the minimum of SPR curve. To determine the sensitivity of the studied sensor structures, we used well known water solutions in a flow-through two-channel flow cell that provided contact between liquid and the structures of the first and second types. The flow-through flow cell was equipped with respective tubes for inflow and outflow of liquid.

The influence of deposition geometry on the sensor sensitivity we studied in the refractometer "Plasmon-71" with laser wave length $850 \mathrm{~nm}$ and flow cell volume 70 micro liter. SPR refractometer "Plasmon-71" (Figure 2) developed at the V. E. Lashkaryov Institute of Semiconductor Physics under the National Academy of Sciences of Ukraine. The sensitivity of refractometer "Plasmon-71" was improved to $4 \times 10^{-6}$ RIU. This device is capable of measuring one sample of the studied substance in an automated mode, displaying the obtained results on the monitor of a computer within 5 - 10 seconds. The optical scheme of the device is depicted in Figure 2(a). Its dimensions are $215 \times 130 \times 100 \mathrm{~mm}$.



Figure 1. Appearance of the vacuum depositing device ВУП-5М. 




(a)

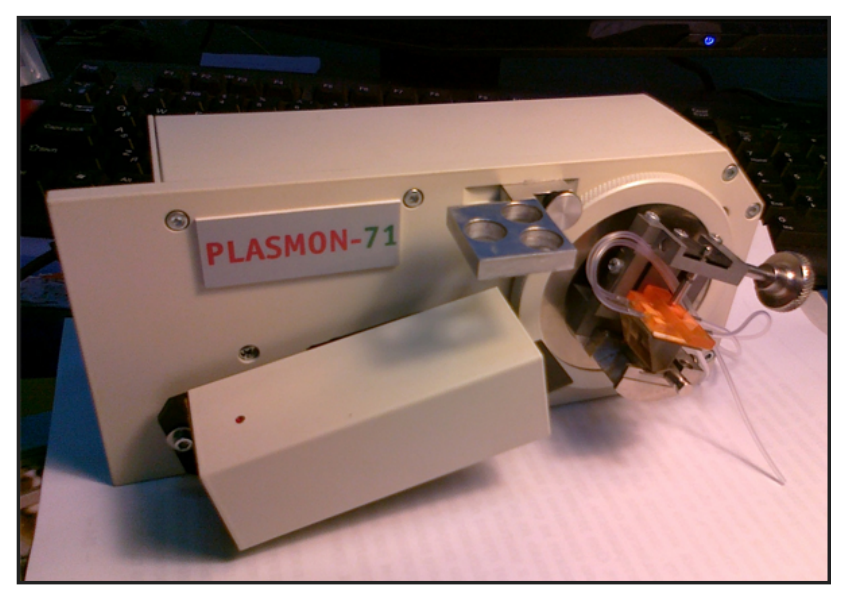

(b)

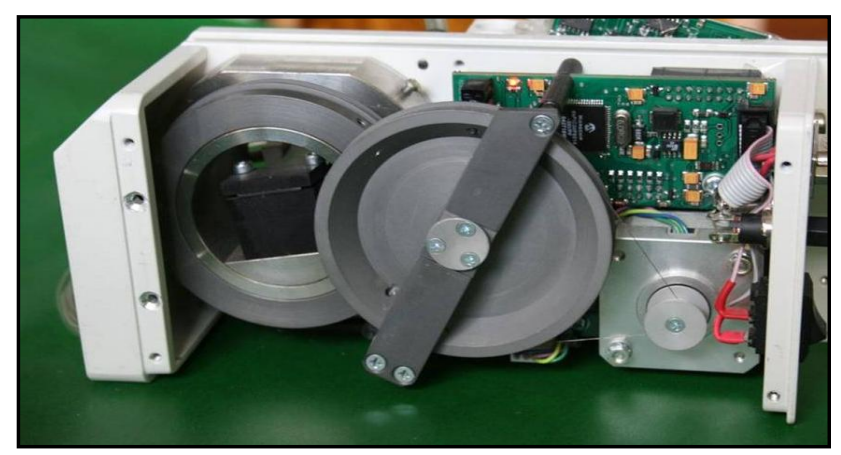

(c)

Figure 2. SPR spectrometer Plasmon-71: the kinematic scheme of the device (a); a view of the front panel of the device with the optical unit, prism and flow-cell (b); the reverse of the front panel on which the board of the electronic block, step motor and reduction system are fixed (c).

As far as the design is concerned, the device is made as a mono-block structure consisting of the two bearing flat plates: the horizontal one and vertical rest, which are T-coupled with each other. The optical unit and measuring flow-cell with a prism are fixed on the one side of the rest, while the board of an electronic block for control and measurements as well as a step motor rotating the prism with a sensor is fixed on the other side of the rest. The optical unit and measuring flow-cell with a prism are depicted in Figure 2(b). The board of the electronic block for control and measurements as well as a step motor rotating the prism is depicted in Figure 2(c).

The rotation of the prism changes the angle of incidence for a monochromatic laser beam striking a prism surface that enables registration of a minimum on the SPR curve (Figure 3 ).

For investigation, we used two liquids with different refractive indexes: distilled water $(n=1.33154)$ and solution of potassium chloride $(\mathrm{KCl})$ in water with different percentage from 2.5 to 20 percent. Substitution of liquid samples was provided with a peristaltic micro-pump (Figure 4). We fixed the change in the refractive index 


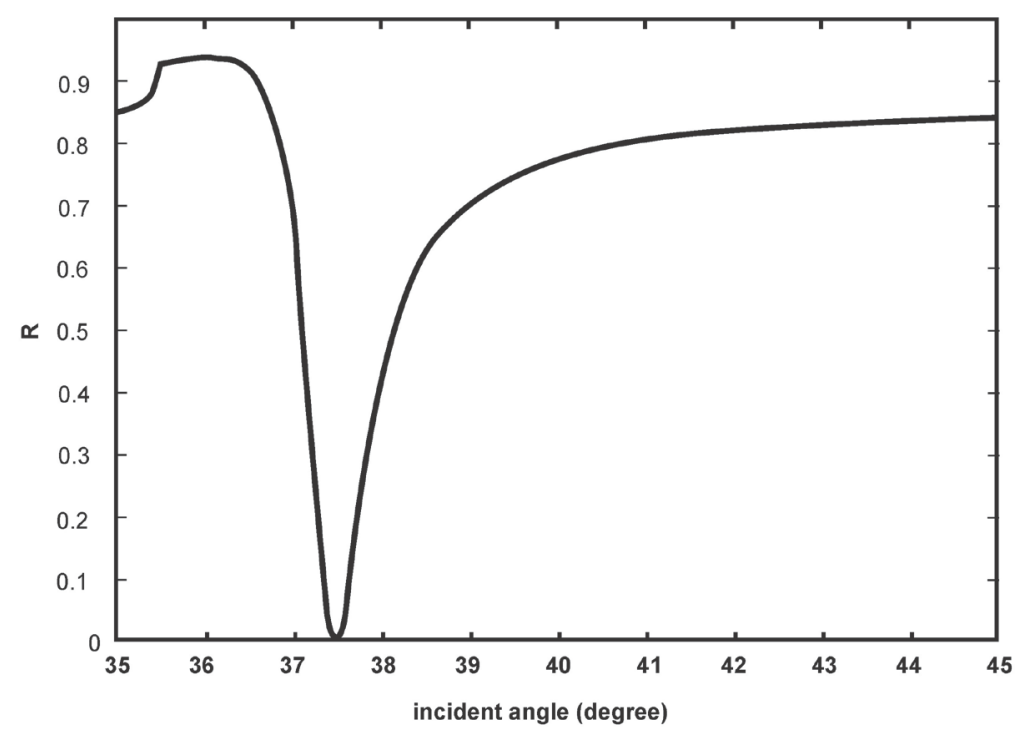

Figure 3. SPR curve.

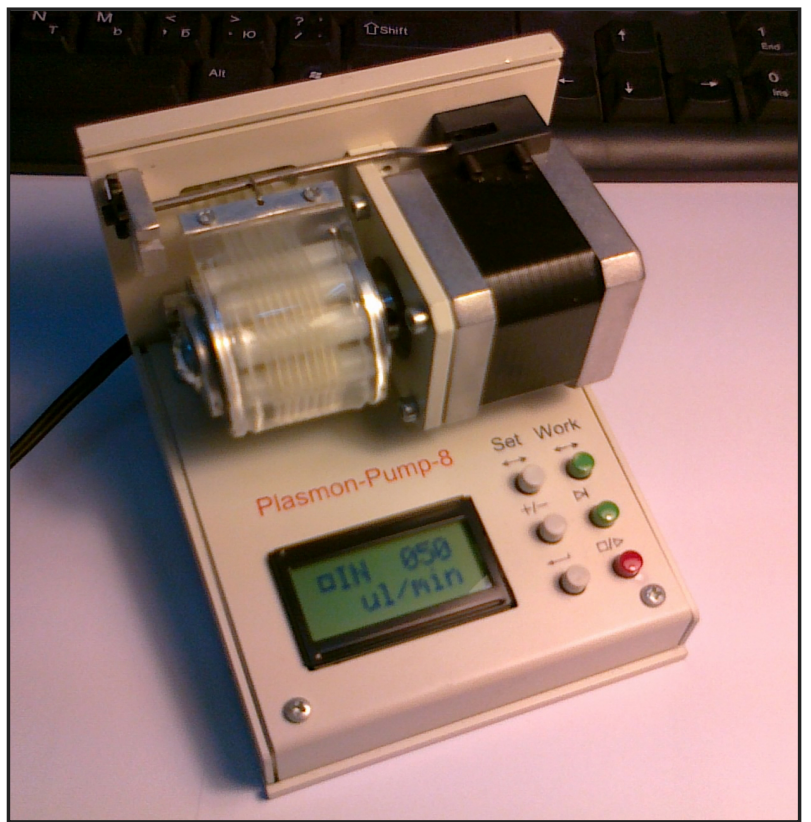

Figure 4. Appearance of the peristaltic micro-pump.

with time at the moment when the flow cell contained distilled water and after substitution of it with salt solution.

The kinetics of the SPR minimum shift was recorded using the special developed program. To decrease possible errors of measurements as a result of oscillations in the ambient temperature, the SPR refractometer "Plasmon-71" as well as flow cells with the studied liquids was placed into a thermostat kept at the temperature $22^{\circ} \mathrm{C}$ $\pm 0.5^{\circ} \mathrm{C}$.

\section{Results}

Topography of the surfaces inherent to the samples of both types was studied using an atomic-force microscope (Figure 5). It was ascertained that the structure of gold layer (Figure 5(b)) prepared using angular deposition with deposition angle 45 degree possesses more developed surface and, respectively, larger area of the surface 




(a)

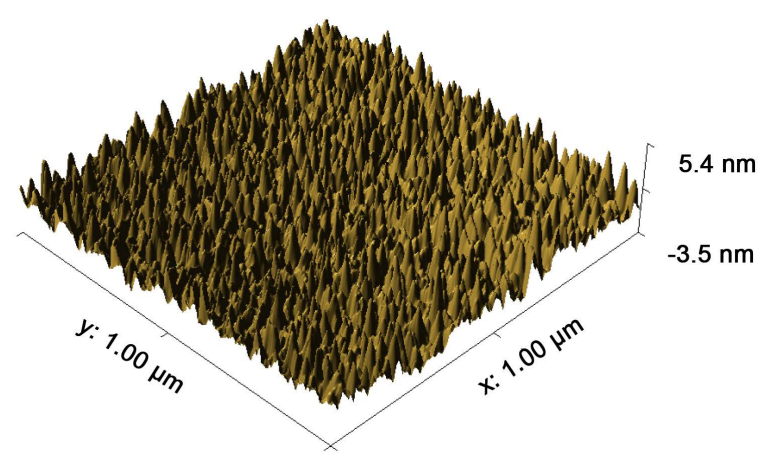

(b)

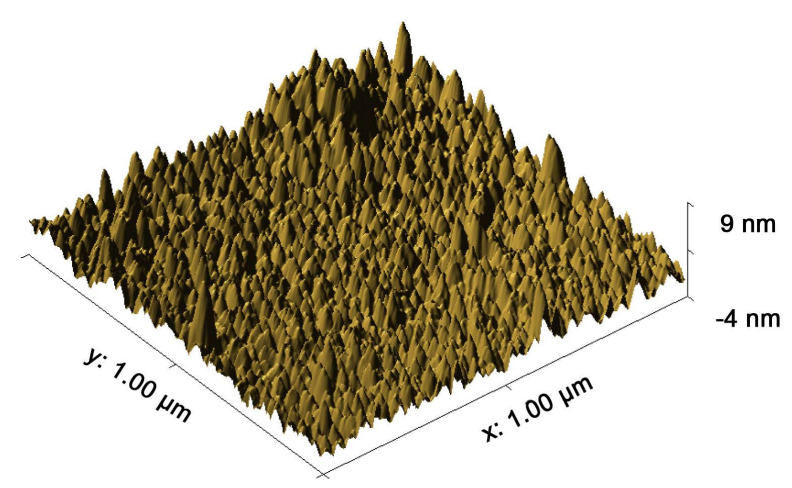

(c)

Figure 5. AFM images of researching sample: structure number 1 (a) and structure number 2 with deposition angle 45 (b) and 70 (c) degree.

as compared to that for the another samples: standard gold sample (Figure 5(a)) and structure of gold layer prepared using angular deposition with deposition angle 70 degree (Figure 5(c)). SPR curves for all samples performance in Figure 6. All SPR curves were measured with refractometer "Plasmon-71".

After for stages of liquid substitution for 24 minutes of measurements, when the type 1 sample was used, the refractive indexes of liquids changed considerably as compared with their initial values (Figure 7, curve blue). In the case of the sample type 2 with deposition angle 45 degree, the refractive index values of distilled water and salt solution remained practically unchanged for the time of measurements (Figure 7, curve red).

\section{Discussion}

The enhancing of the sensitivity of SPR refractometer sensor element is a result of influence of two optical effects: surface plasmon resonance in metal layer and localized surface plasmon resonance between grains tops (Figure 8). 


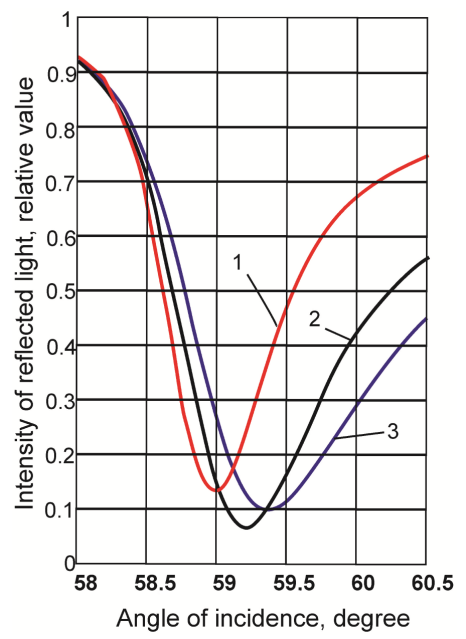

Figure 6. SPR curves of samples: structure number 1(red-1) and number 2 with deposition angle 45 (black-2) and 70 (blue-3) degree.

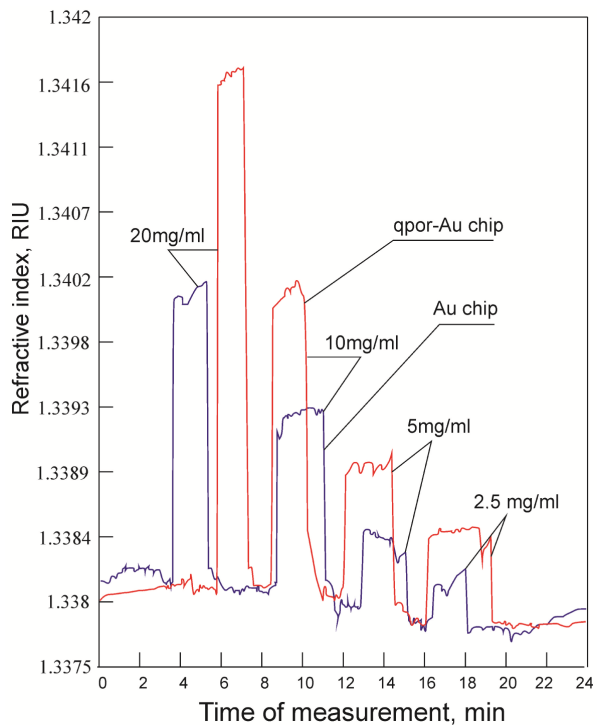

Figure 7. Substitution kinetics for liquids on the surface of sensitive element in the SPR sensor for difference percentage of solution.

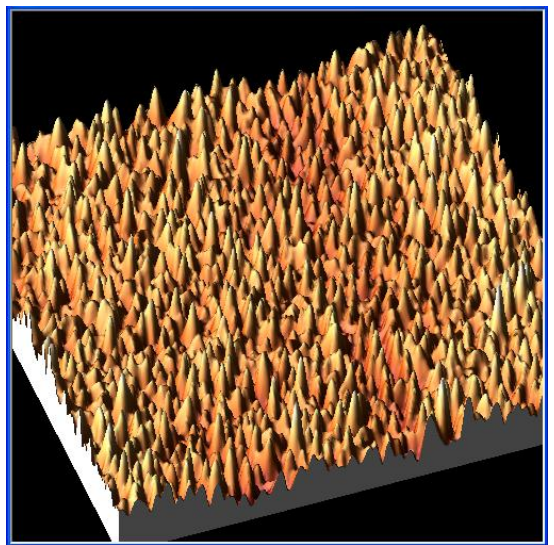

Figure 8. AFM 3-D images of structure number 2 with deposition angle 45 degree. 
Influence of the geometry of the deposition of nanosized gold film of the sensor to improve the results of SPR measurements, perhaps, due to the increase of active points and surfaces interact with the analyte.

\section{Conclusion}

Considered in this paper have been the factors influencing the sensitivity of analytical devices based on surface plasmon resonance. The increase in the sensitivity of measurements is limited by frequency stability and the spectral width of semiconductor laser radiation, photodetector noises and thermal drift of facility. Adduced in the paper have been the results concerning the development of technological solutions aimed at enhancing the sensitivity of SPR devices. It has been found that deposition geometry for the sensitive layer of SPR sensor influences considerably the sensor response to changes in the refractive index of ambient medium. Offered by authors is deposition geometry for gold films of the optimal thickness, which provides 1.4 times higher response to changes in the medium contacting with the sensitive element of SPR sensor.

\section{References}

[1] de Mol, N.J. and Fischer, M.J.E. (2010) Surface Plasmon Resonance: Methods and Protocols (Methods in Molecular Biology). Humana Press, New York.

[2] Homola, J., Yee, S. and Gauglitz, G. (1999) Surface Plasmon Resonance Sensors: Review. Sensors and Actuators B: Chemical, 54, 3-15. http://dx.doi.org/10.1016/S0925-4005(98)00321-9

[3] Kobzeva, I.N. (2009) Optical Sensors for Bio-Sensoric Diagnostical Systems Based on Surface Plasmon Resonance. Herald of KSPU Named by Mykhailo Ostrogradskyi, Issue 4, Part 2.

[4] Ushenin, Yu.V., Khristosenko, R.V., Samoylov, A.V., Dorozinsky, G.W., Kaganovich, E.B., Manoilov, E.G. and Snopok, B.A. (2012) Optoelectronic Sensor Structures Based on Films of Porous Aluminum Oxide Prepared Using Pulsed Laser Deposition. Optoelektronika i Poluprovodnikovaya Tekhnika, No. 47, 40-45.

[5] Ushenin, Yu.V., Khristosenko, R.V., Samoylov, A.V., Kaganovich, E.B., Manoilov, E.G., Snopok, B.A., Dorozinsky, G.V. and Maslov, V.P. (2013) Porous Alumina Films Obtained by pulsed Laser Deposition for Sensor Applications. International Conference "Functional Material" (ICFM-2013), Partenit, 29 September-5 October 2013, 451.

[6] Vakaryuk, T.Ye., Gromovoi, Yu.S., Danko, V.A., Dorozinsky, G.V., Zynio, S.A., Indutnyi, I.Z., Samoylov, A.V., Ushenin, Yu.V., Khristosenko, R.V. and Shepeliavyi, P.Ye. (2013) Application of Porous SiOx Films in Sensors Based on Surface Plasmon Resonance. Optoelektronika i Poluprovodnikovaya Tekhnika, No 48, 89-95.

[7] Palagushkin, A.N., Prokopenko, S.A. and Sergeev, A.P. (2009) Plasmonic Holographic Nanostructures. Optical Memory and Neural Networks, 18, 156-163. http://dx.doi.org/10.3103/S1060992X09030047

[8] Shirshov, Yu.M., Samoylov, A.V., Zinyo, S.A., Surovceva, E.R. and Mirskiy, V. (2002) Bimetallic Layers Increase Sensitivity of Affinity Sensors Based on Surface Plasmon Resonance. Sensors, 2, 62-70. http://dx.doi.org/10.3390/s20200062

[9] Ushenin, Yu.V., Samoylov, A.V. and Khristosenko, R.V. (2011) Enhancing the Sensitivity to Changes in the Medium Refractive Index in Sensors Based on Surface Plasmon Resonance. Tekhnologiya i konstruirovaniye v electronnoi apparature, No 1-2, 12-14.

[10] Dostalek, J., Ctyroky, J., Homola, J., Nekvindova, P., Spikova, J., Skvor, J. and Schrofel, J. (2001) Surface Plasmon Resonance Biosensor Based on Integrated Optical Waveguide. Sensors and Actuators B: Chemical, 76, 8-12. http://dx.doi.org/10.1016/S0925-4005(01)00559-7

[11] www.reichertspr.com

[12] Baiborodin, Yu.V. (1988) Basics of Laser Technique. 2nd Edition, Vyshcha Shkola, Kyiv, 383 p.

[13] Dorozinsky, G., Maslov, V., Samoylov, A. and Ushenin, Yu. (2013) Reducing Measurement Uncertainty of Instruments Based on the Phenomenon of Surface Plasmon Resonance. American Journal of Optics and Photonics, 1, 17-22.

[14] Dorozinsky, G., Dunaevsky, V. and Maslov, V. (2013) Thermal-Vision Method of Investigations and Control of Device Based on Surface Plasmon Resonance. Universal Journal of Control and Automation, 2, 34-39. 